29. Kohlhaw, G. B., Leary, T. R. \& Umbarger, H. E. Alpha-isopropylmalate synthase from Salmonell typhimurium. Purification and properties. J. Biol. Chem. 244, 2218-2225 (1969).

Supplementary Information accompanies the paper on Nature's website ( http://www.nature.com/nature)

Acknowledgements We thank L. Bao, B.-M. Dai, J. Yan, C. Werts, M. Picardeau and G. Baranton for suggestions and comments on our research strategy and manuscript preparation; C. Jin and G.-C. Liu of the Institute of Microbiology, Chinese Academy of Science, for help in the attempt at assaying the enzymatic activity of PafAH; Y. Liu and H.-G. Zhu for help in preparing the drawings; X. Mao and G. Cai for help in computer simulation; B.-Y. Hu and Y-X. Nie for help in bacterial culture preparation; and the members of CHGCS for support and encouragement. This work was supported by the National Natural Science Foundation of China, the Chinese National High Technology Development Program (863), the National Key Program for Basic Research (973) and the Sciences and Technology Commission of the People's Government of Shanghai Municipality. It was also supported by the Pôle Sino-Français en Sciences du Vivant et en Génomique and le Programme de Recherches Avancées Franco-Chinois PRA B00-05.

Competing interests statement The authors declare that they have no competing financial interests.

Correspondence and requests for materials should be addressed to G.-P.Z (e-mail: gpzhao@sibs.ac.cn). The sequences have been submitted to NCBI under accession numbers GB: AE010300 and GB: AE010301 for the large (CI) and small (CII) chromosomes, respectively.

\section{The methylated component of the Neurospora crassa genome}

Eric U. Selker ${ }^{\star}$, Nikolaos A. Tountas $\nmid \neq$, Sally H. Cross $\dagger \neq$, Brian S. Margolin $\star$, Jonathan G. Murphy ${ }^{\star}$, Adrian P. Bird $\dagger$ \& Michael Freitag*

* Department of Biology and Institute of Molecular Biology, University of Oregon, Eugene, Oregon 97403, USA

$\dagger$ Institute of Cell and Molecular Biology, University of Edinburgh, Edinburgh, EH9 3JR, UK

Cytosine methylation is common, but not ubiquitous, in eukaryotes. Mammals ${ }^{1}$ and the fungus Neurospora crass $^{2,3}$ have about $2-3 \%$ of cytosines methylated. In mammals, methylation is almost exclusively in the under-represented $\mathrm{CpG}$ dinucleotides, and most CpGs are methylated ${ }^{1}$ whereas in Neurospora, methylation is not preferentially in CpG dinucleotides and the bulk of the genome is unmethylated ${ }^{4}$. DNA methylation is essential in mammals $s^{5}$ but is dispensable in Neurospora ${ }^{3,6}$, making this simple eukaryote a favoured organism in which to study methylation. Recent studies indicate that DNA methylation in Neurospora depends on one DNA methyltransferase, DIM-2 (ref. 6), directed by a histone $\mathrm{H} 3$ methyltransferase, DIM-5 (ref. 7), but little is known about its cellular and evolutionary functions. As only four methylated sequences have been reported previously in $N$. crassa, we used methyl-binding-domain agarose chromatography $^{8}$ to isolate the methylated component of the genome. DNA sequence analysis shows that the methylated component of the genome consists almost exclusively of relics of transposons that were subject to repeat-induced point mutation-a genome defence system that mutates duplicated sequences ${ }^{9}$.

To isolate the methylated component of the $N$. crassa genome, we cleaved genomic DNA with the 5-methylcytosine-sensitive restriction enzyme Sau3AI (recognition sequence GATC) so as to leave intact patches of methylated DNA, and then passed it over a methylCpG domain (MBD) column, which fractionates according to the

$\ddagger$ Present addresses: Center for Cell Signaling, University of Virginia School of Medicine, Charlottesville, Virginia 22908, USA (N.A.T.); MRC Human Genetics Unit, Western General Hospital, Edinburgh, EH4 2XU, UK (S.H.C.); Department of Biochemistry and Biophysics, University of California San Francisco, California 94143, USA (B.S.M.). degree of CpG methylation ${ }^{8}$. Bound DNA was eluted with increasing concentrations of salt, and fractions were analysed by Southern hybridizations, probing for an unmethylated sequence $(\mathrm{am})$ and previously identified methylated regions $\left(\zeta-\eta, \Psi_{63}\right.$ and ribosomal DNA; Fig. 1). DNA complementary to the am probe eluted principally in pool four but trailed through to pool nine. In contrast, $\zeta-\eta$ sequences peaked later, in pool nine, suggesting that the MBD column successfully fractionated Neurospora DNA on the basis of methylation. Considering that the MBD does not bind methylated non-CpG sites ${ }^{10}$, these findings suggest co-localization of methylated $\mathrm{CpG}$ and non-CpG (Sau3AI) sites. By this assay, the

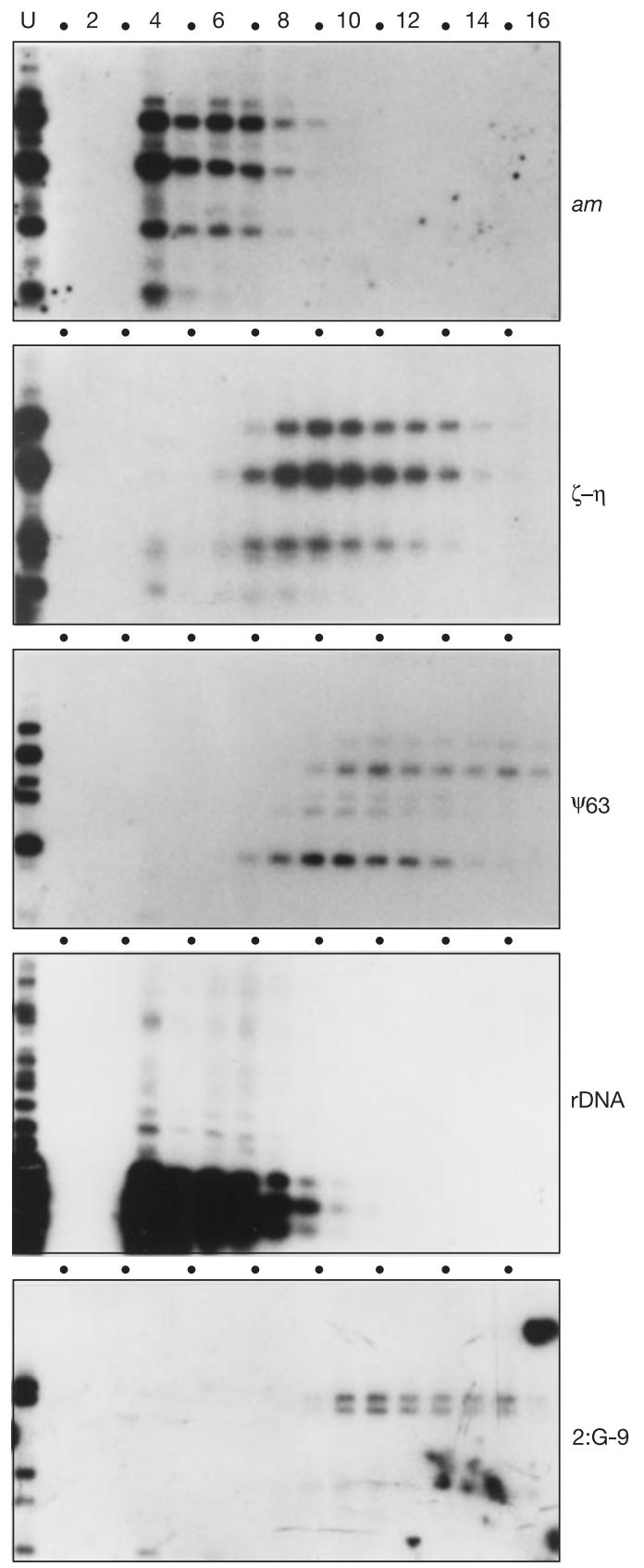

Figure 1 Fractionation of Neurospora DNA on a methylated-DNA-binding column. Samples (about $0.5 \mu \mathrm{g}$ ) of pooled pairs of fractions off the MBD column (1-16 represent column fractions 1-32) were fractionated by agarose gel electrophoresis, along with an unfractionated (U) sample of the SaußAl-digested DNA, blotted to nylon membrane, and probed sequentially for known unmethylated (am) and methylated ( $\zeta-\eta, \Psi_{63}$ and rDNA) sequences, as well as a candidate methylated sequence from this study (2:G-9). 
density of CpG methylation varied within and between sequences. For example, a significant amount of the $\zeta-\eta$ and $\Psi_{63}$ regions of DNA were distributed between pools 4-13, presumably reflecting heterogeneous methylation (Fig. 1). Also, some $\Psi_{63}$ fragments bound more tightly than nearly all the $\zeta-\eta$ DNA, whereas rDNA, which is known to include some methylated cytosines ${ }^{2}$, eluted early, indicating that it has lighter methylation than the two relics of repeat-induced point mutation (RIP).

DNA from tightly bound fractions was cloned and used to probe Southern blots of the MBD-fractionated DNA and/or total genomic DNA digested with MboI and Sau3AI. Some clones (such as 2:G-9; Fig. 1) represented methylated chromosomal regions as expected, but many showed no evidence of methylation, indicating that the MBD column enriched for, but did not fully purify, the methylated sequences (data not shown). We therefore used differential colony hybridization with probes made from early or late column fractions to screen the library for clones that should represent methylated sequences (see Supplementary Fig. 1). DNA was isolated from about 100 of the resulting candidate methylated DNA clones and tested by probing against DNA from a wild type or from the DNA methyltransferase (DMTase) mutant dim-2 (ref. 6) (Fig. 2). The fraction of high-molecular-mass signal in Sau3AI bands was highly variable, consistent with the idea that methylation levels in different chromosomal regions are variable. Results obtained with dim-2 DNA support the conclusion that the DIM-2 DMTase is responsible for all DNA methylation in vegetative cells of Neurospora $a^{6}$. About $70 \%$ of the clones were found to contain sequences that are unambiguously methylated in the Oak Ridge (OR) wild-type strain (source of the DNA). We thus established a set of experimentally validated methylated DNA clones.

Previous work revealed that trichostatin A (TSA), a potent inhibitor of histone deacetylases, causes hypomethylation of RIP (repeat-induced point mutation) copies of $\mathrm{am}$, but does not affect methylation of rDNA and the inactivated transposon Punt ${ }^{R I P 1}$ in the $\Psi_{63}$ region $^{11}$. We used our collection of methylated DNA clones to explore the range of the TSA effect and found only minor consequences (Fig. 3). TSA-induced hypomethylation probably reflects the sensitivity of the DIM-5 HMTase to the acetylation state of lysines in histone $\mathrm{H} 3$ (H. Tamaru and E.U.S., unpublished data), and the differential effects may reflect different activities of histone acetyltransferases and/or histone deacetylases in various chromosomal regions.

We also used the methylated DNA clones to characterize two mutants defective in methylation (dim-1 and dim-3 (refs 12 and 3, respectively)) and to investigate whether the identified methylated sequences are present and methylated in a relatively distant wildtype strain, Mauriceville $(\mathrm{M})^{13}$. Unlike dim-2, neither dim-1 nor dim-3 caused complete loss of methylation; however, they caused

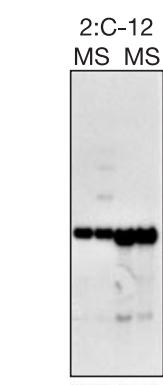

$\operatorname{dim}-2 \overline{+} \overline{-}$
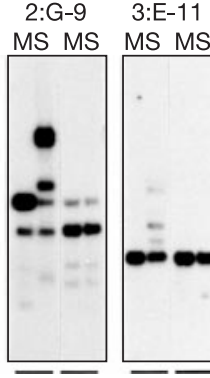

$+-$
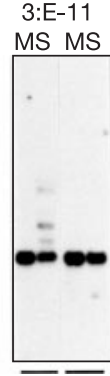

$\overline{+}-$
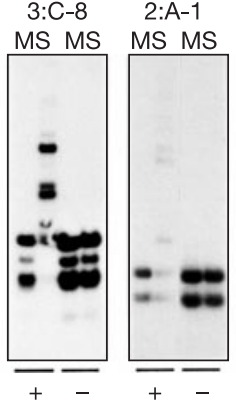

2:A-8 MS MS

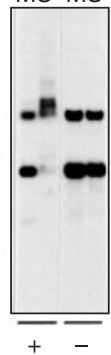

Figure 2 Verification of DNA methylation in genomic DNA regions. Clones representing the indicated potential methylated chromosomal regions were used as probes for Southern blots of Mbol-digested (M) or SaußAl-digested (S) genomic DNA prepared from wild-type $(+)$ or dim-2(-) strains. different patterns of hypomethylation (Fig. 3). Probings of M-DNA revealed many polymorphisms, both in primary sequences and methylation levels, and although many of the sequences are repetitive in both OR and M strains, the levels and patterns of repetition are quite variable. Some of the strongest hybridizing sequences of the OR strain appear absent in the M strain and little or no DNA methylation was observed in the $\mathrm{M}$ strain for many sequences (such as 8:A6 and 8:B9). We conclude that regions of DNA methylation are not highly conserved.

The Neurospora genome has relatively little (approximately 8\%) repetitive $\mathrm{DNA}^{14}$, presumably because of the operation of RIP 9 Notably, nearly $50 \%$ of the methylated clones hybridized to repetitive DNA (data not shown). We sequenced the ends of 50 methylated clones and calculated two 'RIP indices' ${ }^{15}$, based on the observation that RIP preferentially mutates $5^{\prime} \mathrm{CpA}$ : $5^{\prime} \mathrm{TpG}$ to generate TpA dinucleotides. For 38 of 50 methylated DNA clones analysed, both indices were diagnostic for RIP. Four clones were positive for one index only and a further three showed evidence of RIP only after detailed analysis (see below). Only 8 of 50 sequences showed ratios typical of unmutated Neurospora genes, but three of these were too short to calculate the indices reliably. We conclude that the methylated component of the Neurospora genome consists almost exclusively of sequences mutated by RIP.

To gain insight into the natural targets of RIP, we used BLAST ${ }^{16}$ to compare the methylated sequences to sequences in GenBank and $N$. crassa genome and proteome databases (including sequences not assembled; http://www-genome.wi.mit.edu/annotation/fungi/ neurospora/). Notably, 32 of 46 sequences analysed resemble transposons including Punt ${ }^{R I P 1}$ (Table 1, 5:H8 (ref. 15)), gypsylike retrotransposon $d a b-1$ (11:G2 (ref. 17)), the long interspersed nucleotide element (LINE)-like element Tad (11:D3 (ref. 18)), and the copia-like element Tcen (2:C10, 2:H9, 5:D4, 8:F8 (ref. 19)). We also found relics of transposons not previously known in Neuro-
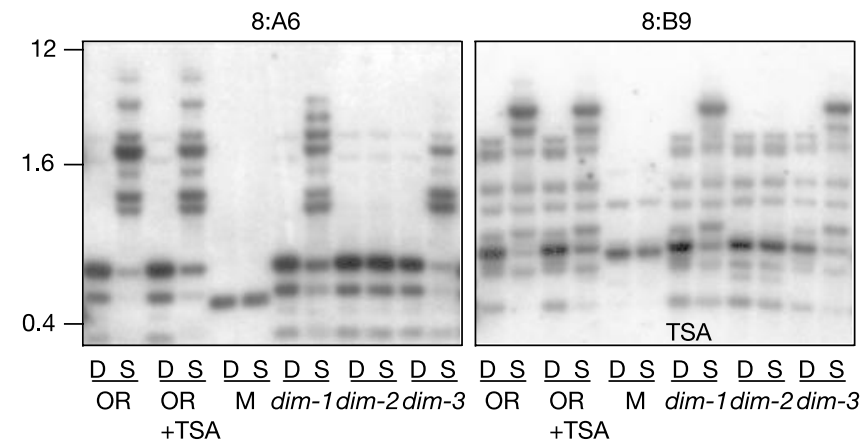

8:C9
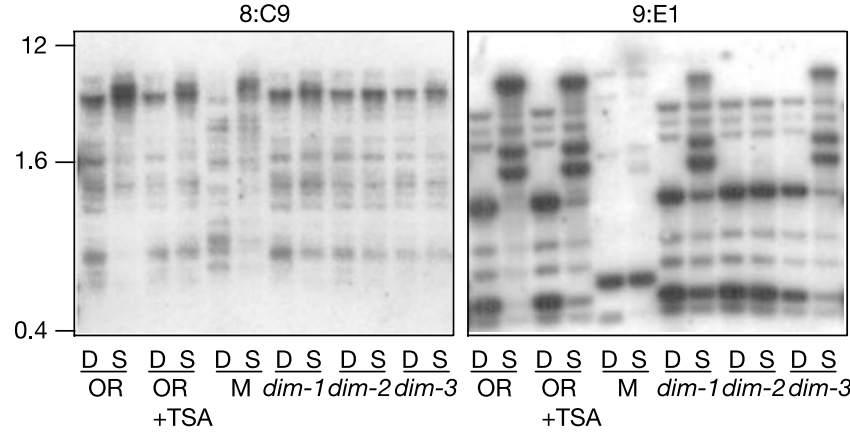

Figure 3 Comparison of DNA methylation in Oak Ridge (OR), Mauriceville (M) and dim strains of $N$. crassa. Approximately $1 \mu \mathrm{g}$ of DNA from OR (FGSC 2489), M (FGSC 2225), dim-1 (N2517), dim-2 (N1104) or dim-3 (N1089) were digested with Dpnll (D) or Sau3AI (S) and used for Southern hybridizations with probes from clones generated in this study. The OR strain was grown in the presence $(+)$ or absence $(-)$ of $1 \mu \mathrm{g} \mathrm{ml}^{-1}$ TSA as previously described ${ }^{11}$. Positions of size standards $(\mathrm{kb})$ are shown on left. Additional examples are presented in Supplementary Fig. 2. 
spora, including the gypsy-like Lolligag (2:A11, 3:C11, Table 1) and DNA-type transposons of the $h A T^{20}$ and Tcl/mariner ${ }^{21}$ superfamily (Table 1; see also Supplementary Fig. 3). No relics of true mariner transposons occur in the Neurospora genome sequence ${ }^{22}$. Several methylated fragments could not be identified unequivocally as relics of transposons but represented RIP-mutated repeated sequences (for example, 2:A1, 3:E11, 4:D12, 5:F8, 9:E1, 11:E5). We also isolated four methylated segments consisting of RIP-mutated rDNA (7:D8, 7:E5, 7:G9, 8:A10, Table 1) whose origin is uncertain. Finally, three of the four Tcen homologues and five additional methylated fragments from centromeric regions provide evidence of DNA methylation in centromeric regions of Neurospora.

What caused the methylation of the rare methylated regions with non-diagnostic RIP indices? Previous studies showed that some relics of RIP can induce methylation de novo and that methylation can extend far from a sequence serving as a methylation signal ${ }^{23}$. Thus, methylation could result from flanking signals. Alternatively, sequences with non-diagnostic RIP indices might nevertheless be products of RIP. Sequence comparisons between the methylated sequences and related sequences in the genome revealed instances of the latter possibility. Two independently isolated, heavily methylated and $(\mathrm{A}+\mathrm{T})$-rich fragments of a sequence related to the Minos transposon in Drosophila (8:A6 and 8:B1, Table 1) provide an example. Comparison with a homologous sequence (contig 3.422 ) revealed 21 and 68 mutations characteristic of RIP, respectively. Similarly, methylated clone 5:A7, which was not predicted to be a product of RIP on the basis of RIP indices (Table 1), contains a RIP-mutated 259-base-pair (bp) segment of the Dodo2 transposon relic.

To test directly for methylation signals within the 5:A7 region, we introduced 5:A7 segments into Neurospora and checked for de novo methylation. We targeted single copies of the full 736-bp fragment, the 451-bp Dodo2 fragment or the adjacent 447-bp sequence, without extraneous (for example, vector) sequences, to the his-3 or am loci and tested for induction of methylation (Supplementary Fig. 4). All fragments induced substantial methylation, indicating that flanking methylation signals are not required. The non-Dodo2 segment of 5:A7 represents a rare example of de novo methylation in

\begin{tabular}{|c|c|c|c|c|c|c|c|c|}
\hline $\begin{array}{l}\text { Clone } \\
\text {................... }\end{array}$ & Accession $^{\star}$ & Coordinates & Linkage group $†$ & Methylation (\%)‡ & Repeats§ & $\frac{T p A}{A p T}$ & $\frac{(\mathrm{CpA}+\mathrm{TpG})}{(\mathrm{ApC}+\mathrm{GpT})}$ & Comments\| \\
\hline 2:A1 & AY227784 & - & - & $\sim 50$ & $\sim 2$ & 1.41 & 0.51 & Best match near Cen-III \\
\hline $2: A 10$ & AABX01000180 & 35973-36621 & III (acr-2) & $\sim 50$ & $\sim 3$ & 1.28 & 0.75 & Fo Folyt1; near Cen-III; Nc Listless \\
\hline 2:A11 & AABX01000358 & $148-2458$ & $\mathrm{VII}(w c-1)$ & $\sim 50$ & $\sim 8$ & 1.40 & 0.36 & Mg MGLR-3; Nc Lolligag \\
\hline 2:B3 & AABX01000083 & $8526-9112$ & I (hsp30) & $\sim 60$ & 1 & 1.26 & 0.91 & Putative RIP-mutated kinase gene (5:D1) \\
\hline 2:C9 & AABX01000208 & $141257-141617$ & IV $($ aod-1) & $\sim 90$ & $\sim 10$ & 1.14 & 1.05 & Nc Punt linked to Nc Punt RIP1 \\
\hline 2:C10 & AABX01000326 & 36860-37830 & VI (cax) & $\sim 90$ & $\sim 30$ & 1.47 & 0.42 & Nc Tcen; near TEL VI L \\
\hline 2:C11 & AY227785 & - & - & $\sim 50$ & $\sim 15$ & 1.27 & 0.64 & Match adjacent to Ant1; Nc Dodo2 \\
\hline 2:C12 & AABX01000364 & $11925-13203$ & VII (arg-10) & $\sim 30$ & $\sim 12$ & 1.38 & 0.63 & Mg Occan; Nc Punt3 \\
\hline 2:E8 & AABX01000505 & 20022-21060 & - & $\sim 50$ & $\sim 12$ & 1.42 & 0.35 & Mg Occan; Nc Punt3 \\
\hline 2:G9 & AABX01000238 & $15504-16798$ & IV (nit-4) & $>90$ & $\sim 5$ & 1.33 & 0.66 & Hs Tigger; Nc Nogo \\
\hline 2:G12 & AABX01000227 & 20529-21108 & $\mathrm{IV}(p y r-1)$ & $\sim 80$ & $\sim 5$ & 1.33 & 0.74 & Hs Tigger; Nc Nogo \\
\hline 2:H9 & AABX01000304 & $27426-28777$ & $V(i l v-2)$ & Trace & $\sim 30$ & 1.13 & 0.90 & Nc Tcen; near Cen-V \\
\hline 3:C11 & AL670001 & $10535-12971$ & $\|(\operatorname{aro}-1)$ & $\sim 70$ & $\sim 8$ & 1.47 & 0.32 & Mg MGLR-3; Nc Lolligag (3:C8) \\
\hline 3:E11 & AABX01000368 & $44939-45532$ & VII (met-7) & $\sim 40$ & 1 & 1.45 & 0.89 & Adjacent to repeats \\
\hline 4:D12 & AY227786 & - & - & $\sim 30$ & $\sim 10$ & 1.23 & 0.45 & Related to RIP-mutated sequences \\
\hline 5:A7 & $\begin{array}{l}\text { AABX01000101 } \\
\text { AABX01000060 }\end{array}$ & $\begin{array}{c}12231-12478 \\
1-477\end{array}$ & I (met-6) & $\sim 50$ & $\sim 25$ & 0.75 & 1.15 & An Ant1; links two contigs; Nc Dodo2 \\
\hline 5:B8 & AL355932 & $41108-43653$ & $\|(g p d-1)$ & $>90$ & $\sim 5$ & 1.42 & 0.59 & Hs Tigger; Nc Nogo (8:E12) \\
\hline 5:C6 & AABX01000442 & 2357-3354 & - & $\sim 5$ & 1 & 0.76 & 1.06 & Adjacent to Mg pth11 homologue \\
\hline $5: C 9$ & AABX01000651 & $755-1022: 1-268$ & - & $\sim 40$ & $\sim 20$ & 1.36 & 0.61 & Adjacent to An Ant1-like transposon \\
\hline 5:D1 & AABX01000083 & 8059-9112 & I (hsp30) & $\sim 40$ & 1 & 1.40 & 0.93 & Near Cen-I (2:B3) \\
\hline 5:D2 & AABX01000425 & 2242-3293 & - & $\sim 50$ & $\sim 30$ & 1.08 & 0.94 & Resembles Nc En/Spm flanks; SNF2-like \\
\hline 5:D4 & AABX01000270 & $3332-4249$ & $\vee(i l v-2)$ & $\sim 20$ & $\sim 30$ & 1.35 & 0.54 & Nc Tcen; near Cen-V \\
\hline 5:D6 & AABX01000110 & 3898-4202 & I (ad-5) & Trace & $\sim 25$ & 1.08 & 0.95 & An Ant1; Nc Dodo2 \\
\hline 5:F8 & AABX01000051 & 4623-6648 & I (cyt-1) & $\sim 50$ & $\sim 9$ & 1.47 & 0.50 & Repeat region \\
\hline 5:H8 & AABX01000241 & $26017-27007$ & IV $(\operatorname{aod}-1)$ & $\sim 40$ & $\sim 10$ & 1.12 & 0.91 & Identical to Nc Punt ${ }^{\text {RIP1 }}$ \\
\hline $6: A 3$ & AABX01000207 & $1412-3547$ & IV $($ con-10) & $\sim 50$ & $\sim 40$ & 1.26 & 0.67 & Adjacent to Aa Quetzal; Nc Dodo1 (8:F5) \\
\hline 6:B8 & AABX01000028 & 82503-86325 & I (vma-11) & $\sim 5$ & $\sim 12$ & 1.43 & 0.56 & Mg Occan; Nc Punt3 \\
\hline 6:D5 & AABX01000436 & $61687-63770$ & - & $30 \rrbracket$ & $\sim 30$ & 1.31 & 0.86 & Within repeats near Cen \\
\hline 7:D8 & AY227787 & - & VL & $\sim 40$ & $\sim 150$ & 0.61 & 0.89 & rDNA\# \\
\hline 7:E5 & AY227788 & - & $\mathrm{VL}$ & $\sim 10$ & $\sim 150$ & 1.00 & 0.86 & rDNA\# \\
\hline 7:G9 & AY227789 & - & $\mathrm{VL}$ & $\sim 15$ & $\sim 150$ & 0.83 & 1.05 & rDNA\# \\
\hline 8:A6 & AABX01000266 & 36323-37403 & $V($ spe-2) & $\sim 90$ & $\sim 12$ & 0.86 & 1.27 & Dh Minos; Nc Dodo1 (8:B1) \\
\hline 8:A10 & AY227790 & - & $V L$ & $\sim 20$ & $\sim 150$ & 0.92 & 1.15 & rDNA\# \\
\hline 8:B1 & AABX01000266 & 34422-37522 & $\mathrm{V}(\mathrm{spe}-2)$ & $>90$ & $\sim 12$ & 0.74 & 1.14 & Dh Minos; Nc Dodo1(8:A6) \\
\hline 8:B9 & AABX01000207 & 61975-62675 & IV (con-10) & $\sim 70$ & $\sim 11$ & 0.82 & 0.96 & Mg Pot3; Nc Punt2 \\
\hline 8:C9 & AABX01000206 & 136650-141576 & IV (pho-5) & $\sim 20$ & $\sim 25$ & 1.39 & 0.52 & An Ant1; Nc Dodo2 \\
\hline 8:E12 & AL355932 & 41108-43659 & $\|(g p d-1)$ & $\sim 30$ & $\sim 5$ & 1.42 & 0.60 & Hs Tigger; Nc Nogo (5:B8) \\
\hline 8:F3 & AL670001 & 20545-22136 & II (aro-1) & $>90$ & $\sim 8$ & 1.44 & 0.54 & gypsy-type relic \\
\hline 8:F5 & AABX01000207 & 1409-3547 & IV $($ con-10) & $\sim 40$ & $\sim 40$ & 1.25 & 0.67 & Adjacent to Aa Quetzal; Nc Dodo1 (6:A3) \\
\hline 8:F8 & AABX01000436 & $38371-40249$ & & $\sim 50$ & $\sim 30$ & 1.56 & 0.52 & Rice helicase homologue; adjacent Nc Tcen \\
\hline 8:F10 & AY251480 & $450914-454187$ & III (mip-1) & $\sim 50$ & 1 & 1.47 & 0.37 & - \\
\hline 8:G3 & AL669988 & $70281-71810$ & II (ro-3) & $>90$ & $\sim 9$ & 1.21 & 0.73 & Nh-like transposase; Nc Dodo3 \\
\hline 9:E1 & AABX01000427 & $71841-73691$ & - & $\sim 90$ & $\sim 4$ & 1.57 & 0.53 & RIP-mutated repeat \\
\hline 11:D3 & AABX01000610 & 2064-2752 & - & 301 & $\sim 60$ & 1.39 & 0.30 & Nc Tad; near Cen \\
\hline 11:E5 & AABX01000204 & 110785-116088 & $\mathrm{IV}(\mathrm{ro}-1)$ & $\sim 60$ & $\sim 8$ & 1.28 & 0.72 & RIP-mutated repeat \\
\hline 11:G2 & AABX01000297 & 35403-36604 & V (spe-3) & $\sim 60$ & $\sim 20$ & 1.78 & 0.47 & Nc dab-1 \\
\hline
\end{tabular}

*GenBank accession numbers for contigs (assembly 3 of WICGR; http://www-genome.wi.mit.edu/annotation/fungi/neurospora/). Accession numbers beginning with 'AL' refer to MIPS sequences (http:// www.mips.biochem.mpg.de/proj/neurospora/). Fragments 2:A1, 2:C11, 4:D12, 7:D8, 7:E5, 7:G9 and 8:A10 had no perfect matches in Assembly 3.

†Linkage group and closest genetic marker (in parentheses).

$\ddagger$ Degree of DNA methylation estimated from Southern hybridizations (data not shown).

$\S$ The number of transposon relic repeats was estimated from database searches. Matches with a cutoff expected value of $1 \times 10^{-4}$ were counted. Given the variability of RIP this may be a conservative estimate.

\|Best matches (for example, to transposons) are shown (independent overlapping clones are indicated in parentheses). Aa, Anopheles albimanus; An, Aspergillus niger; Dh, Drosophila hydei; Fo, Fusarium oxysporum; Hs, Homo sapiens; Mg, Magnaporthe grisea; Nc, Neurospora crassa; Nh, Nectria haematococca. Newly described Neurospora transposon relics include Listless, Lolligag, Nogo, Punt2, Punt3 and Dodo1 to Dodo3.

१ Heterogenous methylation (that is, some bands were unchanged, whereas others were $>90 \%$ methylated).

\#RIP-mutated copies of Neurospora 25S/28S rDNA, perhaps from the rDNA cluster on linkage group V L (similar or identical sequences in clones 7:E5, 7:D8, b2-1, b2-2, 7:D5, 8:A10). 

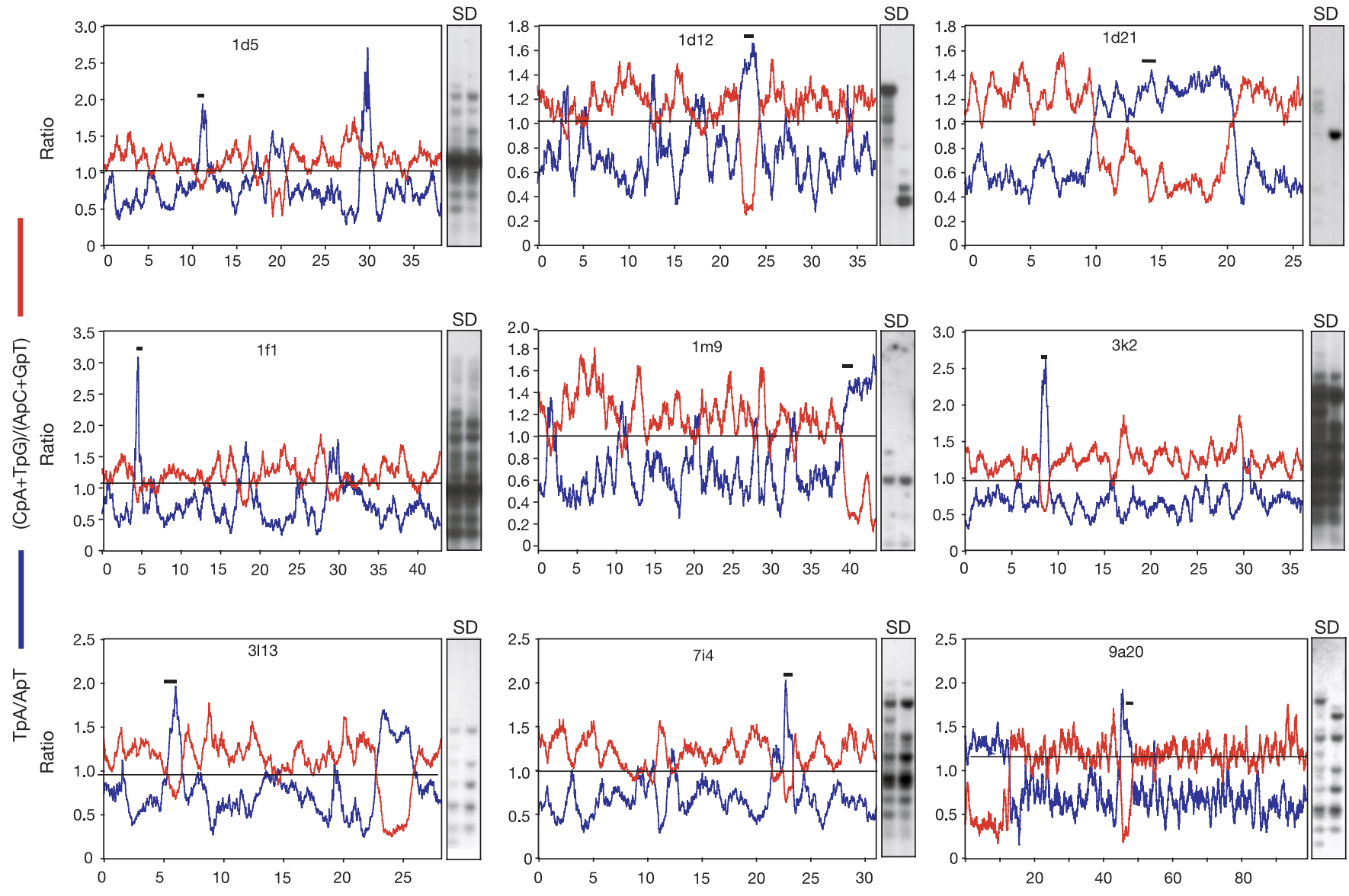

Position (kb)

Figure 4 Methylation of sequences with extreme RIP indices. The dinucleotide composition of $3.7 \mathrm{Mb}$ of non-redundant genomic DNA from linkage group $\mathrm{V}(1 \mathrm{~d} 5,1 \mathrm{~d} 12$, 1d21, 1f1, 1 m9, 714 and 9a20) and VI (3k2 and 3113) (http://www.mips.biochem.mpg.de/ proj/neurospora/) was analysed in $200 \mathrm{bp}$ windows, with $100 \mathrm{bp}$ shifts in $\approx 40-\mathrm{kb}$ segments. Sequences with TpA/ApT ratios $>0.89$ and/or $(\mathrm{CpA}+\mathrm{TpG}) /(\mathrm{ApC}+\mathrm{GpT})$ ratios
$<1.03$ are considered relics of RIP ${ }^{15}$. Representative results from 9/120 contigs analysed are shown. Bars above selected TpA/ApT peaks represent polymerase chain reaction fragments (see Supplementary Table 1) generated to test for methylation of corresponding genomic DNA sequences by Southern hybridization with Sau3Al-(S) or Dpnll-(D) DNA. Resulting autoradiograms are shown to the right of the plots.
Neurospora induced by a sequence showing no evidence of RIP.

Products of RIP are frequently, but not invariably, methylated ${ }^{9}$. To determine whether most natural relics of RIP are methylated, we first calculated RIP indices for sequences of almost an entire Neurospora chromosome (Fig. 4). Most (86 of 120) of the approximately 40-kilobase $(\mathrm{kb})$ segments contained at least one apparent relic of RIP. ((TpA/ApT) $-(\mathrm{CpA}+\mathrm{TpG}) /(\mathrm{ApC}+\mathrm{GpT})>0)$. A total of 174 apparent relics of RIP, ranging from about 0.5 to about $13 \mathrm{~kb}$ (average of about $1.5 \mathrm{~kb}$ ) were found, accounting for approximately $5 \%$ of the genomic sequences examined (roughly 0.2 out of 3.7 megabases $(\mathrm{Mb})$ ). To determine the fraction of putative relics of RIP that are methylated, we performed Southern hybridizations with probes generated for 20 representative sequences. Notably, 19 of 20 showed evidence of methylation (Fig. 4). Thus DNA methylation and relics of RIP are highly correlated in the $N$. crassa genome (see also ref. 22).

Several model eukaryotes, including Saccharomyces cerevisiae, Schizosaccharomyces pombe, Caenorhabditis elegans and Drosophila melanogaster have no or little DNA methylation. At the other extreme, greater than $25 \%$ of cytosines are methylated in DNA of some higher plants ${ }^{24}$. Mammals and Neurospora fall between these extremes. Although the primary function of this modification remains controversial ${ }^{25,26}$, it is known to prevent gene expression in animals, plants and fungi. Loss of DNA methylation has been shown to reactivate transposons in both Neurospora ${ }^{27}$ and plants $^{28,29}$. We show that in Neurospora, methylation is found almost exclusively in relics of transposons inactivated by RIP. Thus, all indications are that the primary, and perhaps exclusive, function of DNA methylation in Neurospora is to control proliferation of transposons, in conjunction with RIP. Although RIP itself may be limited to certain fungi ${ }^{9}$, methylation is primarily associated with transposons in plants ${ }^{30}$, raising the possibility that genome defence was the original function of this epigenetic process ${ }^{25}$. That normal development depends on DNA methylation in some organisms, such as mammals and Arabidopsis, may reflect newer roles that methylation has assumed ${ }^{26}$.

\section{Methods}

\section{Preparation of Neurospora DNA}

A wild-type strain of N. crassa (74-OR23-IVA; Fungal Genetics Stock Center (FGSC) 2489) was grown in stationary liquid Vogel's minimal medium $\mathrm{N}$ at $31^{\circ} \mathrm{C}$ until saturation (3 days). Genomic DNA was isolated ${ }^{23}$ and purified further by phenol/chloroform extractions, $\mathrm{CsCl}$-ethidium bromide equilibrium gradient centrifugation, extraction with isoamyl alcohol and ethanol precipitation. DNA $(12 \mu \mathrm{g})$ was digested for $2 \mathrm{~h}$ with an excess of Sau3AI, extracted with phenol:chloroform:isoamyl alcohol (49:49:2), precipitated, and suspended in $40 \mu \mathrm{l} 10 \mathrm{mM}$ Tris ( $\mathrm{pH} 8.0$ ) in preparation for chromatography.

\section{Enrichment of methylated DNA on MBD columns}

Neurospora DNA digested with Sau3AI $(10 \mu \mathrm{g})$ was fractionated using an MBD column as described $^{8}$. DNA was ethanol-precipitated from pooled pairs of fractions $(2 \mathrm{ml})$, and

aliquots were analysed by gel electrophoresis and Southern hybridization as described ${ }^{23}$. 


\section{Cloning and identification of methylated sequences}

Approximately $25 \%$ of fractions $17-18,19-20,21-24,25-28,29-32$ and $33-36$ were cloned separately into phosphatase-treated, Bam HI-digested pBLUESCRIBE

(Stratagene), and approximately $20 \%$ of each ligation was electroporated into cells of Escherichia coli PKL-F' (recA, lac, mcrA, mcrB1, hsdR2, supE44, galK2, galT22, metB1 [ $F^{\prime}$ proAB, lacI9Z $\triangle M 15$, Tn10]). Representative white colonies on 5-bromo-4-chloro-3 indoyl- $\beta$-D-galactoside (X-Gal) plates were picked (approximately 400 from fractions $21-$ 24; 400 from fractions 25-28; and 100 from each of the other pools) for analysis. Clones were grown in 96-well microtiter dishes with $0.2 \mathrm{ml} \mathrm{LB}$ medium supplemented with $10 \%$ glycerol and $50 \mu \mathrm{g} \mathrm{ml}^{-1}$ ampicillin, and then transferred to $-70{ }^{\circ} \mathrm{C}$ for storage. Clones representing methylated DNA were tentatively identified by colony hybridization by comparing signals resulting from probing replica blots with labelled DNA from column fractions 7-8 (lane 4 in Fig. 1) and 17-22 (lanes 9-11 in Fig. 1). Purified DNA of promising clones was used to probe Sau3AI- or Mbo I-digested Neurospora DNA by Southern hybridization ${ }^{23}$. Inserts were sequenced at the University of Oregon Biotechnology facility.

\section{Computer analyses}

To investigate the occurrence of relics of RIP in the genome, we used the Windows program of the GCG Wisconsin Package (Accelrys) to scan 3.7 Mb of linkage group V and VI sequence from the German Neurospora genome sequencing consortium (http:// www.mips.biochem.mpg.de/proj/neurospora/) for hallmarks of RIP, and plotted the data using Excel software (Microsoft). The Neurospora genome (http://www-

genome.wi.mit.edu/annotation/fungi/neurospora/) and GenBank sequences (http:// www.ncbi.nlm.nih.gov/BLAST/) were searched for matches of 46 sequenced methylated regions (see Table 1) using BLASTN, BLASTX or TBLASTN ${ }^{16}$. Alignments were performed using nucleic acid and protein sequences at the Biology Workbench website (http:// workbench.sdsc.edu/) with ClustalW.

Received 24 December 2002; accepted 14 March 2003; doi:10.1038/nature01564.

1. Bird, A. P. CpG-rich islands and the function of DNA methylation. Nature 321, 209-213 (1986)

2. Russell, P. J., Rodland, K. D., Cutler, J. E., Rachlin, E. M. \& McCloskey, J. A. in Molecular Genetics of Filamentous Fungi (ed. Timberlake, W.) 321-332 (Alan Liss, New York, 1985).

Foss, H. M., Roberts, C. J., Claeys, K. M. \& Selker, E. U. Abnormal chromosome behavior in Neurospora mutants defective in DNA methylation. Science 262, 1737-1741 (1993).

4. Selker, E. U., Fritz, D. Y. \& Singer, M. J. Dense non-symmetrical DNA methylation resulting from repeat-induced point mutation (RIP) in Neurospora. Science 262, 1724-1728 (1993).

5. Li, E., Bestor, T. H. \& Jaenisch, R. Targeted mutation of the DNA methyltransferase gene results in embryonic lethality. Cell 69, 915-926 (1992).

6. Kouzminova, E. A. \& Selker, E. U. Dim-2 encodes a DNA-methyltransferase responsible for all known cytosine methylation in Neurospora. EMBO J. 20, 4309-4323 (2001).

7. Tamaru, H. \& Selker, E. U. A histone H3 methyltransferase controls DNA methylation in Neurospora crassa. Nature 414, 277-283 (2001).

8. Cross, S. H., Charlton, J. A., Nan, X. \& Bird, A. P. Purification of CpG islands using a methylated DNA binding column. Nature Genet. 6, 236-244 (1994).

9. Selker, E. U. Repeat-induced gene silencing in fungi. Adv. Genet. 46, 439-450 (2002).

10. Meehan, R. R., Lewis, J. D. \& Bird, A. P. Characterization of MeCP2, a vertebrate DNA binding protein that binds methylated DNA. Nucleic Acids Res. 20, 5085-5092 (1992).

11. Selker, E. U. Trichostatin A causes selective loss of DNA methylation in Neurospora. Proc. Natl Acad Sci. USA 95, 9430-9435 (1998)

12. Foss, H. M., Roberts, C. J. \& Selker, E. U. Reduced levels and altered patterns of DNA methylation caused by mutations in Neurospora crassa. Mol. Gen. Genet. 259, 60-71 (1998).

13. Metzenberg, R. L., Stevens, J. N., Selker, E. U. \& Morzycka-Wroblewska, E. Identification and chromosomal distribution of $5 \mathrm{~S}$ rRNA genes in Neurospora crassa. Proc. Natl Acad. Sci. USA 82, 2067-2071 (1985)

14. Krumlauf, R. \& Marzluf, G. A. Genome organization and characterization of the repetitive and inverted repeat DNA sequences in Neurospora crassa. J. Biol. Chem. 255, 1138-1145 (1980).

15. Margolin, B. S. et al. A methylated Neurospora 5S rRNA pseudogene contains a transposable element inactivated by RIP. Genetics 149, 1787-1797 (1998).

16. Altschul, S. F. et al. Gapped BLAST and PSI-BLAST: a new generation of protein database search programs. Nucleic Acids Res. 25, 3389-3402 (1997).

17. Bibbins, M., Cummings, N. J. \& Connerton, I. F. DAB1: a degenerate retrotransposon-like element from Neurospora crassa. Mol. Gen. Genet. 258, 431-436 (1998).

18. Cambareri, E. B., Helber, J. \& Kinsey, J. A. Tad1-1, an active LINE-like element of Neurospora crassa. Mol. Gen. Genet. 242, 658-665 (1994).

19. Cambareri, E. B., Aisner, R. \& Carbon, J. Structure of the chromosome VII centromere region in Neurospora crassa: degenerate transposons and simple repeats. Mol. Cell Biol. 18, 5465-5477 (1998).

20. Kempken, F. \& Windhofer, F. The hAT family: a versatile transposon group common to plants, fungi, animals, and man. Chromosoma 110, 1-9 (2001).

21. Plasterk, R. H., Izsvak, Z. \& Ivics, Z. Resident aliens: the Tc1/mariner superfamily of transposable elements. Trends Genet. 15, 326-332 (1999).

22. Galagan, J. E. et al. The genome sequence of the filamentous fungus Neurospora crassa. Nature 422, 859-868 (2003).

23. Miao, V. P., Freitag, M. \& Selker, E. U. Short TpA-rich segments of the zeta-eta region induce DNA methylation in Neurospora crassa. J. Mol. Biol. 300, 249-273 (2000).

24. Paszkowski, J. \& Whitham, S. A. Gene silencing and DNA methylation processes. Curr. Opin. Plant Biol. 4, 123-129 (2001).

25. Yoder, J. A., Walsh, C. P. \& Bestor, T. H. Cytosine methylation and the ecology of intragenomic parasites. Trends Genet. 13, 335-340 (1997).

26. Bird, A. DNA methylation patterns and epigenetic memory. Genes Dev. 16, 6-21 (2002).

27. Zhou, Y., Cambareri, E. B. \& Kinsey, J. A. DNA methylation inhibits expression and transposition of the Neurospora Tad retrotransposon. Mol. Gen. Genet. 265, 748-754 (2001).

28. Miura, A. et al. Mobilization of transposons by a mutation abolishing full DNA methylation in Arabidopsis. Nature 411, 212-214 (2001).
29. Singer, T., Yordan, C. \& Martienssen, R. A. Robertson's Mutator transposons in A. thaliana are regulated by the chromatin-remodeling gene Decrease in DNA Methylation (DDM1). Genes Dev. 15, 591-602 (2001).

30. Tompa, R. et al. Genome-wide profiling of DNA methylation reveals transposon targets of CHROMOMETHYLASE3. Curr. Biol. 12, 65-68 (2002).

Supplementary Information accompanies the paper on Nature's website ( http://www.nature.com/nature)

Acknowledgements We thank F. K. Selker and C. B. Matsen for help with computer analyses; T. Wolfe for technical assistance; G. Mannhaupt for providing some accession numbers; and J. Galagan for comments on the manuscript. E.U.S. acknowledges the hospitality of the Bird laboratory when he initiated this project while on sabbatical, and is thankful to D. Macleod, R. Meehan and F. Antequera for their advice. This work was supported by a US Public Health Service grant to E.U.S. from the National Institutes of Health, and a Senior International Fellowship from the Fogarty International Center of the National Institutes of Health.

Competing interests statement The authors declare that they have no competing financial interests.

Correspondence and requests for materials should be addressed to E.U.S

(e-mail: selker@molbio.uoregon.edu). Sequences are deposited in GenBank. Accession numbers for sequences isolated with the MBD column are listed in Table 1 and accession numbers for the MIPS sequences (Fig. 4) are: AL670542 (1d5), AL451013 (1d12), AL807371 (first 6 kb of 1d21), BX294013 (last $23.55 \mathrm{~kb}$ of 1d21), AL513410 (1f1), AL513411 (1m9), BX295538 (3k2), BX295540 (3113), AL807369 (7i4), BX295539 (9a20).

\section{Cell fusion is the principal source of bone-marrow-derived hepatocytes}

\section{Xin Wang*, Holger Willenbring*, Yassmine Akkari*, Yumi Torimaru*, Mark Foster ${ }^{\star}$, Muhsen Al-Dhalimy*, Eric Lagasse $\dagger$, Milton Finegold $\neq$, Susan Olson* \& Markus Grompe ${ }^{\star}$}

* Department of Molecular and Medical Genetics, Oregon Health \& Science University, Portland, Oregon 97239, USA

$\dagger$ Stem Cells Inc., Palo Alto, California 94304, USA

$\ddagger$ Department of Pathology, Texas Children's Hospital, Houston, Texas 77030, USA

Evidence suggests that haematopoietic stem cells might have unexpected developmental plasticity, highlighting therapeutic potential. For example, bone-marrow-derived hepatocytes can repopulate the liver of mice with fumarylacetoacetate hydrolase deficiency and correct their liver disease ${ }^{1}$. To determine the underlying mechanism in this murine model, we performed serial transplantation of bone-marrow-derived hepatocytes. Here we show by Southern blot analysis that the repopulating hepatocytes in the liver were heterozygous for alleles unique to the donor marrow, in contrast to the original homozygous donor cells. Furthermore, cytogenetic analysis of hepatocytes transplanted from female donor mice into male recipients demonstrated 80,XXXY (diploid to diploid fusion) and 120,XXXXYY (diploid to tetraploid fusion) karyotypes, indicative of fusion between donor and host cells. We conclude that hepatocytes derived form bone marrow arise from cell fusion and not by differentiation of haematopoietic stem cells.

Recent reports have highlighted the broad developmental potential of bone-marrow-derived stem cells, a phenomenon termed 'stem cell plasticity'. Bone marrow contains haematopoietic stem cells (HSCs) ${ }^{2}$ as well as mesenchymal stem cells ${ }^{3}$ and multipotent adult progenitor cells ${ }^{4}$. HSCs produce not only all of the blood lineages, but also skeletal muscle ${ }^{5}$, neurons ${ }^{6}$, cardiac muscle ${ }^{7}$, and pulmonary $^{8}$ and liver epithelium ${ }^{9-11}$. It has been shown that transplantation of HSCs can act as a substitute for hepatocyte transplantation in a murine model of tyrosinaemia, and HSC transplantation can correct this metabolic liver disease ${ }^{1}$. Although this indicates that 